\title{
ВКЛАД ПОЛА, ВОЗРАСТА И ЧУВСТВИТЕЛЬНОСТИ К ПОДКРЕПЛЕНИЮ В СТРУКТУРУ ЛИЧНОСТНЫХ ЧЕРТ И РАСПРОСТРАНЕННЫХ ПСИХОЛОГИЧЕСКИХ ПРОБЛЕМ ДЕТЕЙ И ПОДРОСТКОВ
}

\author{
Е.Р. СЛОБОДСКАЯ
}

\begin{abstract}
${ }^{a}$ Федеральное государственное бюджетное научное учреждение «Научно-исследовательский институт физиологии и фундаментальной медицины», 630117, Россия, Новосибирск, ул. тимакова, 4
\end{abstract}

\section{Резюме}

Связь чувствительности к наказанию и вознаграждению с компонентами иерархической структуры личностных черт и распространенных психологических проблем изучена на представительных выборках детей 2-18 лет $(\mathrm{N}=968)$ и подростков $11-18$ лет $(\mathrm{N}=1543)$ с помощью адаптированных и валидизированных авторами опросников: краткой формы Списка индивидуальных особенностей ребенка (ICID-S) для оценки личностных черт, опросника Сильные стороны и трудности (SDQ) для оценки эмоциональных и поведенческих проблем и опросника чувствительности к подкреплению (SPSRQ). В общем факторном анализе шкал ICID-S и SDQ установлена четырехуровневая структура, сходная с выявленными ранее иерархическими моделями личности и психопатологии. Четырехфакторная модель включала следующие факторы: Организация, Позитивный, Поведенческие проблемы и Интернализация. Коэффициенты конгруэнтности указали на принципиальное сходство факторов, полученных по данным родителей и самоотчетов подростков. Установлено, что пол, возраст и чувствительность к наказанию и вознаграждению, как правило, вносят независимый вклад на разных уровнях иерархии. Чувствительность к вознаграждению вносит существенный вклад в экстернализацию (проблемы с поведением, гиперактивность и невнимательность), а чувствительность к наказанию во многом определяет интернализацию (застенчивость, страхи и эмоциональные симптомы), при этом связь чувствительности к наказанию с интернализацией у старших подростков была сильнее, чем у младших. Кроме того, установлено, что защитное действие чувствительности к наказанию в отношении экстернализации было значимо только у девочек. Эти результаты свидетельствуют о значении мотивационных систем мозга для развития нормальных и аномальных особенностей поведения и эмоционального реагирования.

Ключевые слова: личностные черты, проблемы поведения, эмоциональные проблемы, чувствительность к подкреплению, иерархическая структура, дети, подростки.

Автор благодарна всем участникам исследования. Эта работа была поддержана грантами: РФФИ № 16-06-00022 (сбор данных), РНФ № 16-18-00003 (анализ данных) и РГНФ № 14-0600139 (подготовка статьи). 


\section{Введение}

Значение систем мозга, контролирующих эмоции, мотивации и поведение, для развития личностных черт и распространенных психологических проблем признано с давних пор (Павлов, 1923). Однако в течение многих лет нормальные индивидуально-психологические особенности и проблемное поведение изучали в разных областях науки (Krueger, Tackett, 2003). Исключение составляют исследования развития, где связи между биологически обусловленными особенностями темперамента/личности и проблемами психического здоровья изучали интенсивно, начиная с работ С. Чесс и А. Томаса (1994). В последние два десятилетия исследователи достигли согласия относительно классификации индивидуально-психологических особенностей на протяжении жизни и возрос интерес к взаимосвязям личности и психопатологии (см. обзоры: Caspi, Shiner, 2008; De Pauw, Mervielde, 2010; Krueger, Tackett, 2003; Tackett, 2006).

Предложено несколько моделей, объясняющих связи личностных черт и распространенных проблем психического здоровья (Caspi, Shiner, 2008; Tackett, 2006), однако многие из них неточны, делают сходный прогноз и не объясняют причинных основ этих связей; предполагается, что исследования развития могут прояснить многие неясные вопросы (Durbin, Hicks, 2014). Однако возрастные изменения личностных черт и выраженности отклонений можно оценить только после того, как будет установлено постоянство структуры личности и психо- патологии в процессе развития. В последние годы показана устойчивость пятифакторной модели личности (Markon et al., 2005); два фактора высшего порядка: Альфа, включающий уступчивость, сознательность и нейротизм, и Бета, включающий экстраверсию и открытость опыту, систематически воспроизводятся в разных странах у людей разного возраста и пола независимо от источника информации (DeYoung, 2006; Digman, 1997; Slobodskaya, 2011). Показано принципиальное сходство иерархической структуры личности у детей и подростков из разных стран с дефинитивной структурой взрослых (Tackett et al., 2012).

Установлено, что распространенная психопатология распределена непрерывно, размерности разного уровня относительно стабильны, два фактора высшего порядка: экстернализация, включающая деструктивность, агрессию, гиперактивность и антисоциальное поведение, и интернализация, включающая тревогу, страхи, депрессивные признаки и психосоматические проблемы, устойчиво выявляются в разных странах в широком возрастном диапазоне (Krueger, Markon, 2006; Lahey et al., 2008). Поскольку распространенные психологические проблемы часто сочетаются и сосуществуют с нормальными личностными чертами, изучение их общей структуры обоснованно (Krueger, Markon, 2006; Krueger, Tackett, 2003). В недавнем исследовании установлено, что иерархическая структура личности и распространенной психопатологии сходна в подгруппах обоего пола от раннего детского до старшего подросткового возраста (Slobodskaya, 
2014), однако остается неясным, зависит ли эта структура от источника информации. Данные родителей являются основным источником информации о младших детях, с возрастом все большее значение приобретают самоотчеты; при этом согласованность данных разных информантов в психопатологии развития обычно невысока и нет общего мнения о том, как лучше их интегрировать (Dirks et al., 2012).

Связи между личностными чертами и распространенной психопатологией установлены в многочисленных исследованиях, однако их природа остается неясной (Tackett, 2006; Caspi, Shiner, 2008). Предполагается, что их общей биологической основой являются системы мозга, контролирующие эмоции, мотивации и поведение (Bijttebier et al., 2009). В теории Грея (1987), в последующем названной теорией чувствительности к подкреплению (Corr, 2008), предполагается, что причиной личностных особенностей и распространенной психопатологии являются индивидуальные различия нейропсихологических систем, чувствительных к вознаграждению и наказанию. Побудительная система вознаграждения вызывает положительные эмоции, связанный с фрустрацией гнев и импульсивное поведение приближения; показано, что она связана с экстраверсией и экстернальными (поведенческими) проблемами. Психофизиологическая система наказания вызывает негативные эмоции страха и тревоги, избегание и торможение текущего поведения; она связана с нейротизмом и интернальной психопатологией (Corr, 2008; Corr et al., 2013). Несмотря на установленные взаимосвязи между показателями чувствительности к подкреплению и характеристиками личности и психопатологии, исследование биологических основ личностных черт и распространенных психологических проблем находится на начальной стадии (Bijttebier et al., 2009; Corr et al., 2013).

Цель настоящей работы - изучение чувствительности к подкреплению как общей основы нормальных личностных особенностей и проблемного поведения. Были поставлены следующие задачи: 1) установить иерархическую структуру личностных черт и распространенных психологических проблем в процессе развития; 2) оценить воспроизводимость этой структуры по данным разных респондентов; 3) определить вклад чувствительности к вознаграждению и наказанию в иерархически организованные конструкты личности и психопатологии; 4) выявить взаимодействие чувствительности к подкреплению с полом и возрастом.

\section{Методы}

\section{Участники}

Исследование 1. Выборка включала 968 детей и подростков (53\% девочек) от 2 до 18 лет ( $\mathrm{M}=10.6, \mathrm{SD}=$ 3.9). Для изучения возрастных различий выборку разделили на четыре группы: дошкольники (2-6 лет, $\mathrm{N}=147)$, младшие школьники (7-10 лет, $\mathrm{N}=341)$, младшие подростки (1114 лет, $\mathrm{N}=288)$ и старшие подростки (15-18 лет, $\mathrm{N}=192)$. Данные получены с помощью родительских опросников, бо́льшая часть респондентов 
(84\%) - матери, 10\% - отцы, остальные - близкие ребенку взрослые. Большинство детей и подростков (68\%) проживали в семьях с двумя биологическими родителями; $20 \%-$ с одним родителем (как правило, с матерью) и 10\% - в сводных семьях (как правило, с матерью и отчимом), остальные - с другими воспитателями; средний размер семьи - четыре человека. Среднее образование имели 10\% матерей и 15\% отцов; среднее специальное - 42\% матерей и $46 \%$ отцов, высшее - 48\% матерей и $39 \%$ отцов. Профессиональное положение родителей варьировало от неквалифицированных работников ручного труда до высококвалифицированных специалистов и руководителей; 18\% матерей и 6\% отцов не работали.

Исследование 2. Выборка включала 1543 подростка (53\% девочек) от 11 до 18 лет; младшие подростки (11-14 лет) составили 35\% выборки. Данные получены с помощью опросников для самоотчетов подростков. Большинство подростков (63\%) проживали в семьях с двумя биологическими родителями; $22 \%$ - с одним родителем (как правило, с матерью) и 12\% - в сводных семьях (как правило, с матерью и отчимом), остальные - с другими воспитателями; средний размер семьи - четыре человека. Среднее образование имели $13 \%$ матерей и 15\% отцов; среднее специальное - 38\% матерей и $42 \%$ отцов, высшее - 49\% матерей и 43\% отцов. Профессиональное положение родителей варьировало от неквалифицированных работников ручного труда до высококвалифицированных специалистов и руководителей; 14\% матерей и 5\% отцов не работали.

\section{Инструментьь}

В исследовании использованы международные опросники для родителей и подростков, адаптированные и валидизированные автором. Надежность шкал оценивали коэффициентом внутренней согласованности $\alpha$ (альфа Кронбаха).

Личностные особенности оценивали краткой формой Списка индивидуальных особенностей ребенка (ICID-S; Slobodskaya, Zupančič, 2010) - свободного от влияния возраста и культуры инструмента, созданного в рамках пятифакторной модели личности. ICID-S содержит 62 краткие характеристики, с помощью которых оценивают 15 личностных черт: активность, антагонизм, боязливость, застенчивость, интеллект/обучаемость, общительность, организованность, ориентацию на достижения, отвлекаемость, открытость опыту, отрицательные эмоции, покладистость, положительные эмоции, сочувствие и упрямство. При валидизации русской версии ICID-S установлены инвариантность пятифакторной структуры опросника в подгруппах различного пола и возраста и хорошая согласованность шкал (Knyazev et al., 2008). В данной работе средний коэффициент внутренней согласованности шкал по данным родителей составил 0.78 (размах 0.68-0.84), по данным подростков - 0.74 (размах 0.64-0.81).

Психологические проблемь оценивали с помощью стандартизованного опросника Сильные стороны и трудности (SDQ; Goodman et al., 2005), предназначенного для измерения проблем психического здоровья распространенных поведенческих и 
эмоциональных трудностей. SDQ содержит 20 утверждений, с помощью которых оценивают выраженность эмоциональных симптомов, проблем с поведением, гиперактивности/невнимательности и проблем со сверстниками. SDQ разработан на основе действующих классификаций поведенческих и психических расстройств и факторного анализа, имеет удовлетворительные психометрические характеристики и широко применяется в разных странах мира. Русская версия SDQ адаптирована и валидизирована в рандомизированной стратифицированной выборке школьников.

Чувствительность к подкреплению оценивали с помощью соответствующих опросников для родителей (SPSRQ-C; Кузнецова, Слободская, 2010) и подростков (SPSRQ-J; Кузнецова и др., 2013). SPSRQ-C содержит 33 утверждения и валидизирован в лаборатории на основе физиологических показателей (Colder et al., 2011); SPSRQ-J содержит 30 вопросов. При валидизации русских версий опросников с помощью конфирматорного факторного анализа подтвердилась двухфакторная структура, включающая шкалы чувствительности к вознаграждению (ЧВ) и чувствительности к наказанию (ЧН). В данной работе коэффициенты внутренней согласованности шкал ЧВ составили 0.81 по данным родителей и 0.70 по данным подростков; шкал ЧН - 0.82 по данным родителей и 0.80 по данным подростков.

Статистический анализ. Иерархическую структуру личностных черт и распространенных психологических проблем изучали с помощью общего факторного анализа шкал ICID-S и SDQ (Goldberg, 2006; Markon et al., 2005). Наибольшее количество факторов устанавливали с помощью параллельного анализа и MAР-теста (O’Connor, 2000). Воспроизводимость факторов в двух исследованиях оценивали с помощью коэффициента конгруэнтности после вращения варимакс и целенаправленного вращения (Fischer, Fontaine, 2011). Вклад пола, возраста и чувствительности к наказанию и вознаграждению в иерархическую структуру личности и психопатологии изучали с помощью корреляционного анализа и общих линейных моделей (General Linear Model). Анализ проводили при помощи IBM SPSS Statistics, версия 22.

\section{Результаты}

Иерархическая структура личностных черт и распространенных психологических проблем у детей $u$ подростков. Анализ общей корреляционной матрицы шкал ICID-S и SDQ выявил пять факторов, удовлетворяющих критерию Кайзера (с собственными значениями $>$ 1) в обоих исследованиях. Параллельный анализ показал, что собственные значения превышали полученные случайным образом только у первых четырех факторов; MAР-тест тоже указал на четырехфакторное решение. Поскольку параллельный анализ и МАР-тест - наиболее обоснованные методы установления количества факторов (O’Connor, 2000), в обоих исследованиях на низшем уровне иерархии было извлечено четыре фактора. Коэффициенты конгруэнтности между оптимально 
подобранными факторами по данным родителей и подростков варьировали от 0.84 до 0.98 , средний коэффициент составил 0.91 , что указывает на воспроизводимость факторов на всех уровнях иерархии. После целенаправленного вращения коэффициенты конгруэнтности факторов варьировали от 0.85 до 0.98, средний коэффициент составил 0.94, что указывает на высокое сходство двух структур. На рисунке 1 представлены иерархические связи между выше- и нижележащими факторами (корреляции 0.30 и выше) и первые пять шкал, определяющие каждый фактор, по данным исследования 1.

Общий фактор на высшем уровне в обоих исследованиях с наибольшими положительными весами вклю- чал покладистость, ориентацию на достижения и организованность наряду с другими положительными чертами (сочувствие, положительные эмоции и интеллект) и с отрицательными весами - отвлекаемость наряду с психопатологическими шкалами. Этот фактор отражает желательные характеристики детей и низкий уровень проблем. На уровне 2 фактор 1 - Экстернализация,по данным родителей, был противоположен по содержанию общему фактору. Фактор 2 с наибольшими положительными весами включал общительность, открытость опыту и активность наряду с интеллектом и положительными эмоциями и с отрицательным весом - застенчивость. Этот фактор принципиально

Рисунок 1

Иерархическая структура личности и психопатологии на основе общего факторного анализа шкал ICID-S и SDQ (данные родителей). Показаны корреляции между ниже- и вышележащими факторами. ОФ - общий фактор; (-) - отрицательная нагрузка

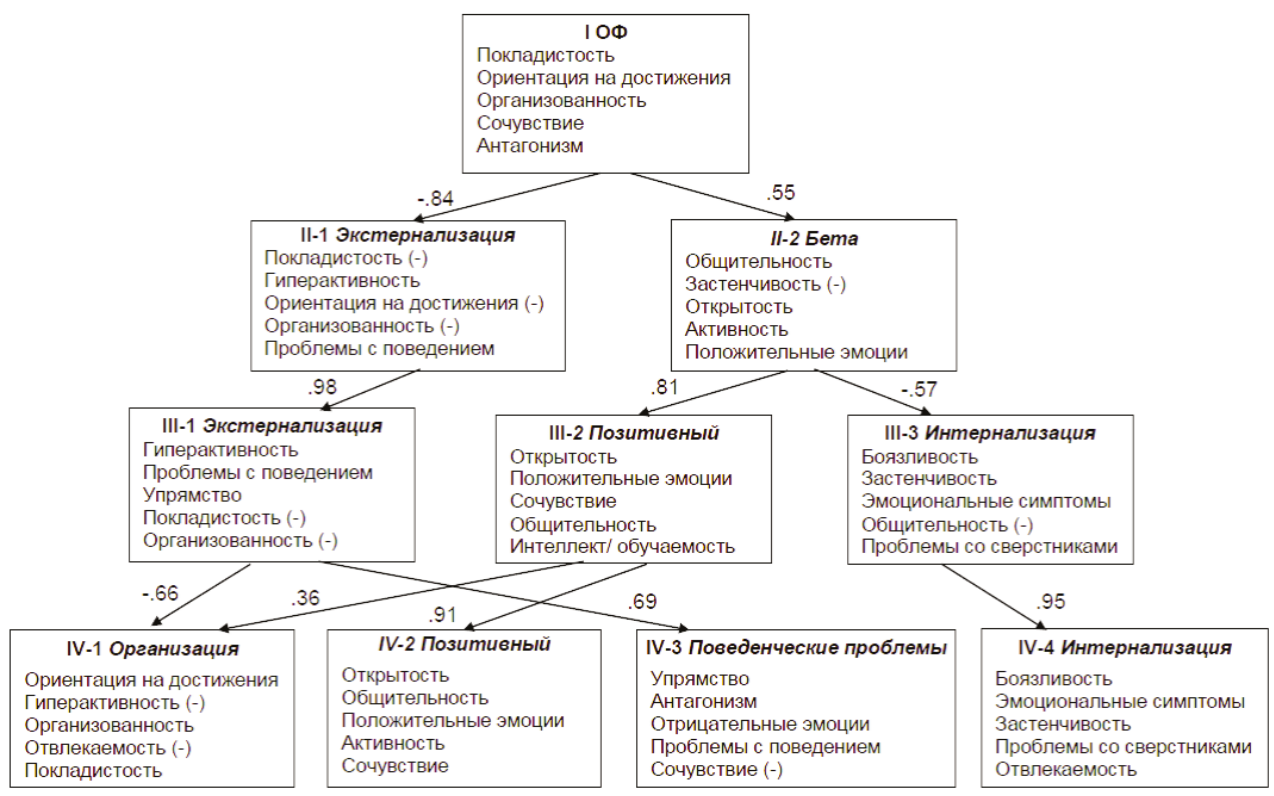


сходен с личностным суперфактором Бета, называемым Пластичность (DeYoung, 2006), а также Личностный рост vs Ограниченность (Digman, 1997). По данным подростков, Бета был ближе к общему фактору $(\mathrm{r}=0.84)$, чем Экстернализация $(\mathrm{r}=$ $=-0.54)$.

На уровне 3 в обоих исследованиях Бета разделялся на Позитивный фактор, включавший ориентацию на достижения, покладистость, сочувствие, положительные эмоции, открытость, общительность, интеллект/обучаемость и активность, и Интернализацию, включавшую застенчивость, боязливость и эмоциональные симптомы, а также общительность с отрицательным весом. Фактор 1, по данным родителей, и фактор 2, по данным подростков, представляли более дифференцированную размерность Экстернализации. На уровне 4 в обоих исследованиях Экстернализация разделялась на Проблемы с поведением, включавшие упрямство, антагонизм, отрицательные эмоции и проблемы с поведением, и фактор Организация, включавший ориентацию на достижения, интеллект, покладистость и организованность, а также отвлекаемость и гиперактивность с отрицательными весами. Факторы Интернализация и Позитивный были практически идентичны соответствующим факторам вышележащего уровня.

Связь чувствительности $\kappa$ подкреплению со структурой личности и nсихопатологии. В таблице 1 даны корреляции между чувствительностью к наказанию и к вознаграждению и факторами личности и психопатологии на четырех уровнях иерархической структуры. В обоих исследованиях чувствительность к наказанию была отрицательно связана с общим фактором и положительно связана с Интернализацией на уровнях 3 и 4. Чувствительность к вознаграждению была положительно связана с фактором Бета на уровне 2 и с Позитивным фактором на уровнях 3 и 4, а также с факторами Экстернализация и Проблемы с поведением. Частичные корреляции показывают, что связи чувствительности к подкреплению с факторами личности и психопатологии мало зависели от пола, возраста и чувствительности к другому виду подкрепления.

Значение пола и возраста. В предшествующем исследовании была показана идентичность иерархической структуры личности и психопатологии у детей и подростков разного пола от дошкольного до старшего подросткового возраста (Slobodskaya, 2014); это дает возможность сравнить средние значения факторов в подгруппах разного пола и возраста. В таблице 2 представлены результаты GLM-анализа, оценивающие вклад пола и возраста в факторы личности и психопатологии на четырех уровнях иерархической структуры. Различия факторов, связанные с полом и возрастом, не зависели друг от друга. Межполовые различия были невелики: в обоих исследованиях пол определял менее $3 \%$ разнообразия факторов на всех уровнях, за исключением четвертого уровня в исследовании 2, где вклад пола в целом превышал 9\%. Направление различий было во многом сходным в обоих исследованиях: у девочек были выше значения общего и позитивного фактора, а у мальчиков - 
выше значения экстернализации и поведенческих проблем. Возрастные различия определяли не более $4 \%$ разнообразия факторов на всех уровнях иерархии. В исследовании 1 значения факторов экстернализации, поведенческих проблем и позитивного фактора у дошкольников и младших школьников были выше, чем у подростков, а интернализации ниже. В исследовании 2 значения факторов экстернализации и Бета у старших подростков были выше, чем у младших.

Помимо главных эффектов, при GLM-анализе в исследовании 2 выявлены взаимодействия чувствительности к подкреплению с полом и возрастом. Пол взаимодействовал с чувствительностью к наказанию в отношении фактора экстернализации на третьем уровне иерархиче-

Таблица 1

Корреляции чувствительности к подкреплению с факторами личности и психопатологии

\begin{tabular}{|c|c|c|c|c|}
\hline \multirow{2}{*}{ Факторы } & \multicolumn{2}{|c|}{$\begin{array}{c}\text { Чувствительность к } \\
\text { наказанию }^{\text {a }} \\
\end{array}$} & \multicolumn{2}{|c|}{$\begin{array}{c}\text { Чувствительность к } \\
\text { вознаграждению }^{\text {b }}\end{array}$} \\
\hline & $\begin{array}{c}\text { Данные } \\
\text { родителей }\end{array}$ & $\begin{array}{c}\text { Данные } \\
\text { самоотчетов }\end{array}$ & $\begin{array}{c}\text { Данные } \\
\text { родителей }\end{array}$ & $\begin{array}{c}\text { Данные } \\
\text { самоотчетов }\end{array}$ \\
\hline I Общий фактор & $\begin{array}{c}-0.25^{* * *} \\
\left(-0.24^{* * *}\right)\end{array}$ & $\begin{array}{l}-0.26^{* * *} \\
\left(-0.28^{* * *}\right)\end{array}$ & $\begin{array}{c}-0.14^{* * *} \\
\left(-0.11^{* * *}\right)\end{array}$ & $\begin{array}{l}0.15^{* * *} \\
\left(0.15^{* * *}\right)\end{array}$ \\
\hline II Экстернализация & $\begin{array}{c}-0.01 \\
(-0.04)\end{array}$ & $\begin{array}{c}0.18^{* * *} \\
\left(0.22^{* * *}\right)\end{array}$ & $\begin{array}{c}0.38^{* * *} \\
\left(0.36^{* * *}\right)\end{array}$ & $\begin{array}{c}0.10^{* * *} \\
\left(0.14^{* * *}\right)\end{array}$ \\
\hline II Бета & $\begin{array}{c}-0.47^{* * *} \\
\left(-0.52^{* * *}\right)\end{array}$ & $\begin{array}{c}-0.20^{* * *} \\
\left(-0.19^{* * *}\right) \\
\end{array}$ & $\begin{array}{c}0.32^{* * * *} \\
\left(0.39^{* * *}\right)\end{array}$ & $\begin{array}{c}0.24^{* * * *} \\
\left(0.26^{* * *}\right)\end{array}$ \\
\hline III Экстернализация & $\begin{array}{c}-0.06 \\
\left(-0.10^{* *}\right)\end{array}$ & $\begin{array}{l}-0.04 \\
(0.02)\end{array}$ & $\begin{array}{c}0.44^{* * *} \\
\left(0.42^{* * *}\right)\end{array}$ & $\begin{array}{c}0.23^{* * *} \\
\left(0.25^{* * *}\right)\end{array}$ \\
\hline III Позитивный & $\begin{array}{l}-0.16^{* * *} \\
\left(-0.19^{* * *}\right)\end{array}$ & $\begin{array}{c}-0.03 \\
(-0.03)\end{array}$ & $\begin{array}{c}0.25^{* * *} \\
\left(0.25^{* * *}\right) \\
\end{array}$ & $\begin{array}{c}0.14^{* * * *} \\
\left(0.15^{* * *}\right) \\
\end{array}$ \\
\hline III Интернализация & $\begin{array}{c}0.58^{* * *} \\
\left(0.59^{* * *}\right) \\
\end{array}$ & $\begin{array}{c}0.56^{* * *} \\
\left(0.55^{* * *}\right) \\
\end{array}$ & $\begin{array}{c}-0.09^{* *} \\
\left(-0.16^{* *}\right)\end{array}$ & $\begin{array}{c}-0.31 * * * \\
\left(-0.31^{* * *}\right) \\
\end{array}$ \\
\hline IV Организация & $\begin{array}{c}0.01 \\
(0.02) \\
\end{array}$ & $\begin{array}{c}-0.13^{* * *} \\
\left(-0.14^{* * *}\right)\end{array}$ & $\begin{array}{c}-0.21^{* * *} \\
\left(-0.19^{* * *}\right)\end{array}$ & $\begin{array}{c}-0.11^{* * *} \\
\left(-0.15^{* * *}\right)\end{array}$ \\
\hline IV Позитивный & $\begin{array}{c}-0.28^{* * *} \\
\left(-0.33^{* * *}\right)\end{array}$ & $\begin{array}{c}-0.01 \\
(-0.00)\end{array}$ & $\begin{array}{c}0.36^{* * *} \\
\left(0.38^{* * *}\right) \\
\end{array}$ & $\begin{array}{c}0.17^{* * *} \\
\left(0.20^{* * *}\right) \\
\end{array}$ \\
\hline $\begin{array}{l}\text { IV Поведенческие } \\
\text { проблемы }\end{array}$ & $\begin{array}{c}-0.09^{* *} \\
\left(-0.12^{* * *}\right)\end{array}$ & $\begin{array}{l}-0.13^{* * *} \\
\left(-0.08^{* *}\right)\end{array}$ & $\begin{array}{c}0.30 * * * \\
(0.30 * * *)\end{array}$ & $\begin{array}{c}0.20^{* * *} \\
\left(0.20^{* * *}\right)\end{array}$ \\
\hline IV Интернализация & $\begin{array}{c}0.54^{* * *} \\
\left(0.54^{* * *}\right)\end{array}$ & $\begin{array}{c}0.61^{* * * *} \\
\left(0.61^{* * *}\right)\end{array}$ & $\begin{array}{c}0.02 \\
(-0.03)\end{array}$ & $\begin{array}{c}-0.30^{* * *} \\
\left(-0.30^{* * *}\right)\end{array}$ \\
\hline
\end{tabular}

Примечание. В скобках даны частичные корреляции: ${ }^{\text {a } ~ с ~ у ч е т о м ~ п о л а, ~ в о з р а с т а ~ и ~ ч у в с т в и т е л ь-~}$

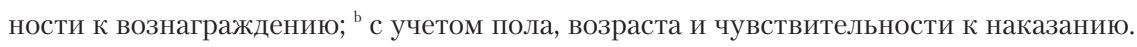

${ }^{*} p<0.05, * * p<0.01, * * * p<0.001$. 
ской структуры, $\mathrm{F}(1,1536)=4.15$; $p<0.05$ : защитное действие чувствительности к наказанию было значимо только у девочек (рисунок 2А). Возраст оказывал модерирующее влияние на связь чувствительности к наказанию с интернализацией на третьем, $\mathrm{F}(1,1536)=13.85 ; p<0.001$, и четвертом уровне иерархии, F (1, $1536)=11.51 ; p=0.001$ : предрасполагающее влияние чувствительности к наказанию у старших подростков было сильнее, чем у младших (рисунок 2Б).

\section{Обсуждение}

Проведенные исследования предоставили сведения об иерархической структуре личностных черт и распространенных психологических проблем, оцененных в представительных выборках по данным родителей детей 2-18 лет и самоотчетов подростков 11-18 лет с помощью валидизированных стандартизованных инструментов. Выявленная в результате общего факторного анализа шкал ICID-S и SDQ четырехуровневая структура согласуется с полученными ранее результатами анализа родительских данных в России (Slobodskaya, 2014), а также с иерархическими моделями личности и психопатологии (Markon et al., 2005; Tackett, 2006). Результаты анализа конгруэнтности указали на принципиальное сходство факторов, полученных по данным родителей и самоотчетов подростков. При этом бо́льшая часть разнообразия оценок в данных родителей объяснялась факторами экстернализации и организации, а в данных подростков суперфактором Бета и Позитивным фактором. Вполне возможно, что это обусловлено разной перспективой респондентов: родители большее

Таблица 2

Вклад пола и возраста в факторы личности и психопатологии на 1-4 уровнях иерархической структуры

\begin{tabular}{|c|c|c|c|c|}
\hline \multirow{3}{*}{ Факторы } & \multicolumn{4}{|c|}{ Пол ${ }^{\mathrm{a}}$} \\
\hline & \multicolumn{2}{|c|}{ Данные родителей } & \multicolumn{2}{|c|}{ Данные самоотчетов } \\
\hline & $F(1,966)$ & $\eta^{2}$ & $F(1,1541)$ & $\eta^{2}$ \\
\hline I Общий фактор & $19.74^{* * *}$ & $0.020($ Д>M) & $24.06^{* * *}$ & 0.015 (Д>M) \\
\hline II Экстернализация & $18.90^{* * *}$ & $0.019(\mathrm{M}>$ Д $)$ & 0.02 & 0.000 \\
\hline II Бета & 2.14 & 0.002 & $32.78^{* * *}$ & 0.021 (Д>M) \\
\hline III Экстернализация & $15.39^{* * *}$ & $0.016(\mathrm{M}>Д)$ & 0.69 & 0.000 \\
\hline ІІІ Позитивный & $7.36^{* *}$ & 0.008 (Д>М) & $43.01^{* * *}$ & $0.027($ Д>M) \\
\hline III Интернализация & 0.01 & 0.000 & 1.58 & 0.001 \\
\hline IV Организация & $25.97^{* * *}$ & 0.026 (Д>M) & $28.69^{* * *}$ & 0.018 ( $\mathrm{M}>$ Д) \\
\hline IV Позитивный & 1.08 & 0.001 & $78.17^{* * *}$ & 0.048 (Д>M) \\
\hline IV Поведенческие проблемы & 1.18 & 0.001 & $25.33^{* * *}$ & $0.016(\mathrm{M}>$ Д $)$ \\
\hline IV Интернализация & 0.24 & 0.000 & $13.77^{* * *}$ & 0.009 (Д>M) \\
\hline
\end{tabular}


Таблица 2 (окончание)

\begin{tabular}{|c|c|c|c|c|}
\hline \multirow{3}{*}{ Факторы } & \multicolumn{4}{|c|}{ Возраст $^{\mathrm{b}}$} \\
\hline & \multicolumn{2}{|c|}{ Данные родителей } & \multicolumn{2}{|c|}{ Данные самоотчетов } \\
\hline & $\mathrm{F}^{\mathrm{e}}$ & $\eta^{2}$ & $\mathrm{~F}(1,1541)$ & $\eta^{2}$ \\
\hline I Общий фактор & 1.52 & 0.005 & 0.20 & 0.000 \\
\hline II Экстернализация & $6.35^{* * *}$ & $\begin{array}{c}0.019 \\
(2-10>11-18)\end{array}$ & $40.59 * * *$ & $\begin{array}{c}0.026 \\
(15-18>10-14)\end{array}$ \\
\hline II Бета & $15.20 * * *$ & $\begin{array}{c}0.045 \\
(2-6>7-18,7- \\
10>15-18)\end{array}$ & $12.08^{* *}$ & $\begin{array}{c}0.008 \\
(15-18>10-14)\end{array}$ \\
\hline III Экстернализация & $9.25^{* * *}$ & $\begin{array}{c}0.028(2- \\
10>11-18)\end{array}$ & $54.11 * * *$ & $\begin{array}{c}0.034 \\
(15-18>10-14)\end{array}$ \\
\hline III Позитивный & $6.06^{* * *}$ & $\begin{array}{c}0.019 \\
(2-6>7-18 \\
7-10>15-18)\end{array}$ & $6.25^{*}$ & $\begin{array}{c}0.004 \\
(15-18>10-14)\end{array}$ \\
\hline III Интернализация & $7.09 * * *$ & $\begin{array}{c}0.022 \\
(2-6<7-18)\end{array}$ & 1.21 & 0.001 \\
\hline IV Организация & $7.98^{* * *}$ & $\begin{array}{c}0.024 \\
(7-10<2-6 \\
11-18)\end{array}$ & $4.43^{*}$ & $\begin{array}{c}0.003 \\
(10-14>15-18)\end{array}$ \\
\hline IV Позитивный & $13.06^{* * *}$ & $\begin{array}{c}0.039 \\
(2-6>7-18 \\
7-10>11-18)\end{array}$ & $7.32^{* *}$ & $\begin{array}{c}0.005 \\
(15-18>10-14)\end{array}$ \\
\hline IV Поведенческие проблемы & $5.11^{* *}$ & $\begin{array}{c}0.016 \\
(2-6>7-18)\end{array}$ & $50.05^{* * *}$ & $\begin{array}{c}0.031 \\
(15-18>10-14)\end{array}$ \\
\hline IV Интернализация & $5.76^{* *}$ & $\begin{array}{c}0.018 \\
(2-6<7-18)\end{array}$ & 1.36 & 0.001 \\
\hline
\end{tabular}

Примечание. ${ }^{\text {a } Н а п р а в л е н и е ~ д о с т о в е р н ы х ~ э ф ф е к т о в, ~ н а п р и м е р, ~ Д ~>~ М ~ о з н а ч а е т, ~ ч т о ~ о ц е н к и ~}$ девочек выше, чем оценки мальчиков. ${ }^{b}$ Направление достоверных эффектов в возрастных группах 2-6, 7-10, 11-14 и 15-18 лет.

${ }^{*} p<0.05,{ }^{* *} p<0.01,{ }^{* * *} p<0.001$.

значение придают упорядоченному поведению, а подростки - положительным устремлениям.

Выявленные в настоящем исследовании четыре фактора: Организация, Позитивный, Поведенческие проблемы и Интернализация - сходны с представленными в литературе четырехфакторными моделями, включающими Сознательность, Уступчивость и Нейротизм Большой Пятерки, а также более широкую размерность Положительной эмоциональности, охватывающую Экстраверсию и Открытость. Следует отметить, что в исследованиях детей 
Рисунок 2

Связь чувствительности к наказанию с факторами личности и психопатологии у подростков разного пола (А) и возраста (Б). Данные самоотчетов
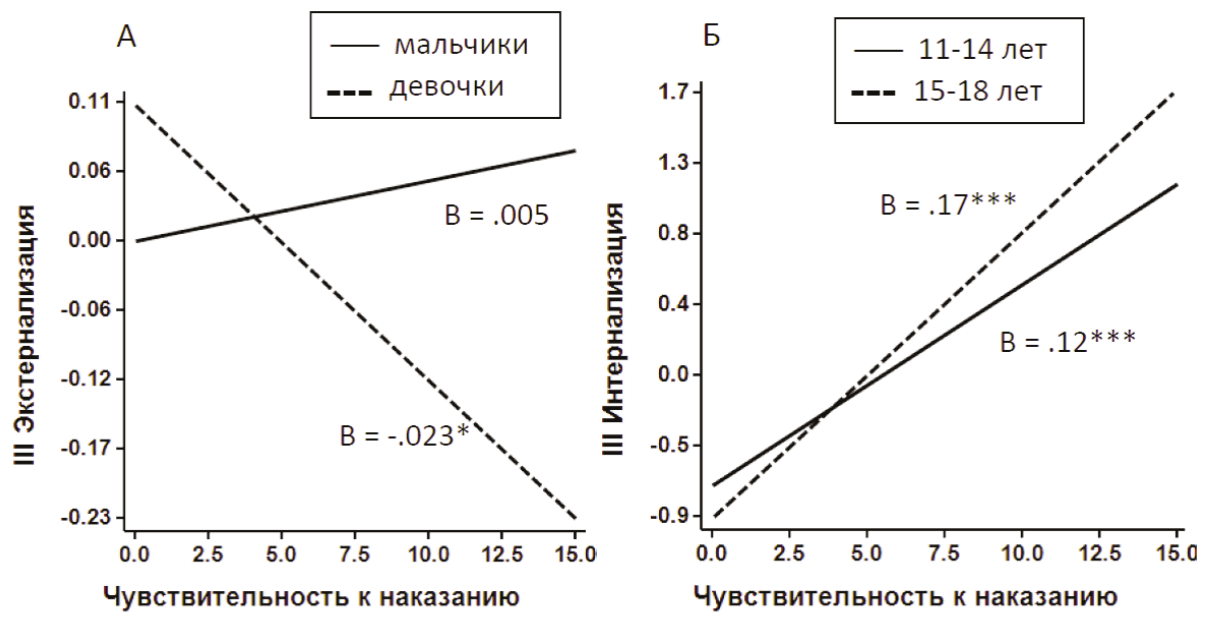

Примечание. В - нестандартизованный регрессионный коэффициент.

${ }^{*} p<0.05,{ }^{* * *} p<0.001$.

и аномального поведения взрослых обычно находят четырехфакторную структуру, возможно потому, что пятый фактор - Открытость - проявляется наиболее поздно и не связан с распространенной психопатологией (Krueger, Tackett, 2003). Примечательно также, что на четвертом уровне иерархии все проблемные шкалы дали существенные нагрузки на три из четырех факторов.

В общей иерархической структуре личностных черт и распространенных психологических проблем фактор Экстернализация появляется на более высоком уровне, раньше дифференцируется и объясняет больше разнообразия, чем фактор Интернализация. В исследованиях взрослых наблюдается обратная кар- тина (Krueger, Markon, 2006; van der Heijden et al., 2013). Это может объясняться тем, что поведенческие проблемы являются наиболее распространенной формой психопатологии у детей и подростков, но к концу подросткового возраста и далее на протяжении жизни их уровень снижается (Tremblay, 2010); эмоциональные проблемы, такие как тревога и депрессия, появляются у старших детей и с возрастом нарастают (Rutter et al., 2008). Предполагается, что внешние наблюдатели, в том числе и родители, являются наилучшим источником информации о поведенческих отклонениях, но значительно хуже отмечают интернальные проблемы и внутренние переживания (Rutter et al., 2008; Tackett et al., 2012), однако в настоя- 
щем исследовании самоотчеты подростков хорошо согласуются с данными родителей в отношении иерархического положения экстернализации и интернализации.

Вклад чувствительности к подкреплению на высших уровнях иерархической структуры личности и психопатологии установлен впервые. Полученные результаты в отношении общего фактора, находящегося на вершине иерархии, в целом соответствуют данным единственного исследования вклада мотивационных системы мозга в общий фактор личности (Erdle, Rushton, 2010), однако в наших исследованиях, включавших проблемное поведение, чувствительность к наказанию вносила больший вклад, чем чувствительность к вознаграждению, а, по данным родителей, чувствительность к вознаграждению была отрицательно связана с общим фактором. Результаты анализа на нижележащих уровнях в обоих исследованиях подтвердили имеющиеся сведения о том, что чувствительность к вознаграждению вносит существенный вклад в экстернализацию, а чувствительность к наказанию во многом определяет интернализацию (Bijttebier et al., 2009; Corr, 2008). Вклад чувствительности к вознаграждению в суперфактор Бета и позитивные факторы установлена впервые, но соответствует теоретическим и эмпирическим данным о связи системы приближения с положительными эмоциями и мотивациями (Corr, 2008; Corr et al., 2013).

Значение пола и возраста для выраженности факторов личности и психопатологии также оценено впервые, однако полученные результаты согласуются с имеющимися данными о большей выраженности экстернальных проблем у лиц мужского пола и о преобладании интернальных проблем у лиц женского пола, по данным самоотчетов (Rutter et al., 2008). Возрастные различия в выраженности экстернализации и интернализации, по данным родителей, соответствуют имеющимся в литературе сведениям о течении распространенных психологических отклонений: выраженность поведенческих проблем снижается от дошкольного до взрослого возраста, а эмоциональные проблемы (тревога и депрессия) нарастают (Rutter et al., 2008; Tremblay, 2010). При анализе взаимодействий установлено, что чувствительность к наказанию и вознаграждению, пол и возраст, как правило, вносят независимый вклад в факторы личности и психопатологии на разных уровнях иерархии. Однако выявленные взаимодействия заслуживают внимания.

Связь чувствительной к наказанию системы поведенческого торможения с последующей интернализацией обнаруживают с раннего детства (Bijttebier et al., 2009; Corr, 2008; Hirshfeld-Becker et al., 2003), наши данные свидетельствуют о том, что эта связь с возрастом усиливается. Возрастание вклада биологических факторов в факторы личности и психопатологии по мере взросления соответствует устоявшимся данным об увеличении роли генетических факторов в поведенческих чертах с возрастом. Так, в метаанализе показано, что наследуемость проявлений тревоги и депрессии, а также экстернального поведения на протяжении подросткового и молодого взрослого 
возраста увеличивается (Bergen et al., 2007). В литературе имеются противоречивые сведения о значении чувствительности к наказанию для экстернализации (Bijttebier et al., 2009; Hundt et al., 2008); полученные результаты указывают на то, что неоднозначные данные могут быть связаны с модерирующим влиянием пола: психофизиологическая система наказания оказывает защитное действие только у лиц женского пола. Для расшифровки этих эффектов необходимы дальнейшие исследования.

\section{Заключение}

В работе исследован вклад чувствительности к наказанию и вознаграждению, пола и возраста в иерархическую структуру личностных черт и распространенных психологических проблем у детей 2-18 лет, по данным родителей и самоотчетов подростков. Выявлено модерирующее влияние пола и возраста на связь чувствительности к подкреплению с поведенческими и эмоциональными проблемами. Эти результаты свидетельствуют о значении мотивационных систем мозга для развития нормальных и аномальных особенностей поведения и эмоционального реагирования. В будущих исследованиях следует учесть ограничения данной работы. Прежде всего это касается использования опросников для оценки чувствительности к подкреплению: желательно привлечь психофизиологические методы хотя бы в небольшой выборке. Кроме того, в каждом исследовании был использован только один источник информации, желательно привлекать нескольких информантов. И, наконец, срезовые исследования позволяют лишь предполагать пути развития, в будущем желательно применять лонгитюдный подход.

\section{Литература}

Грей, Дж. (1987). Нейропсихология эмоций и структура личности. Журнал высшей нервной деятельности, 37(6), 1011-1024.

Кузнецова, В. Б., Слободская, Е. Р. (2010). Опросник для изучения чувствительности к наказанию и вознаграждению у детей. Психологический журнал, 31(2), 145-154.

Кузнецова, В. Б., Слободская, Е. Р., Риппинен, Т. О. (2013). Опросник для изучения чувствительности к наказанию и вознаграждению у подростков. Психологический журнал, 34(1), 69-80.

Павлов, И. П. (1923). Двадцатилетний опыт объективного изучения высшей нервной деятельности (поведения) животных. М./Пг.: Госиздат.

Чесс, С., Томас, А. (1994). Значение темперамента для психиатрической практики. В кн. Е. Р. Слободская (ред.), Детство идеальное и настоящее: Сборник работ современных западных ученых (с. 111-122). Новосибирск: Сибирский хронограф.

Ссылки на зарубежные источники см. в разделе References после англоязычного блока. 


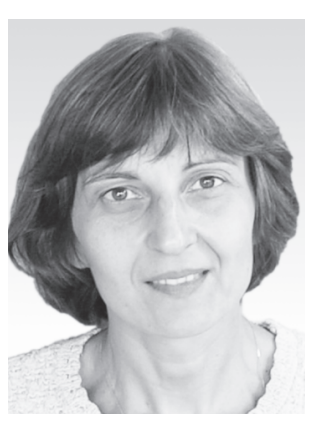

Слободская Елена Романовна - главный научный сотрудник, Федеральное государственное бюджетное научное учреждение «Научно-исследовательский институт физиологии и фундаментальной медицины», доктор психологических наук, доцент.

Сфера научных интересов: развитие личности, психическое здоровье детей.

Контакты: hslob@physiol.ru

\title{
The Contribution of Gender, Age, and Reinforcement Sensitivity to the Structure of Personality Traits and Problem Behaviors in Children and Adolescents
}

\author{
Helena R. Slobodskaya ${ }^{a}$ \\ ${ }^{a}$ Federal State Budgetary Scientific Institution «Scientific Research Institute of Physiology and Basic \\ Medicine», 4 Timakova Str., Novosibirsk, 630117, Russian Federation
}

\begin{abstract}
The questionnaire study examined the contribution of gender, age and reinforcement sensitivity to the common structure of child personality and problem behaviors in two samples, parent reports of 2-18-year-olds $(\mathrm{N}=968)$ and self-reports of 11-18-year-olds $(\mathrm{N}=1543)$, using the Inventory of Child Individual Differences - Short version (ICID-S) to measure personality, the Strengths and Difficulties Questionnaire (SDQ) to assess emotional and behavioral problems, and the Sensitivity to Punishment and Sensitivity to Reward Questionnaire (SPSRQ). A joint factor analysis of the ICID-S and SDQ scales suggested a four-factor structure, including Organization, Positive, Behavior Problems, and Internalizing. This four-factor model was remarkably similar to previously reported models of personality and psychopathology. Congruence coefficients indicated that factors based on parent reports and adolescent selfreports were highly similar at all levels of the hierarchy. Gender, age, sensitivity to punishment, and sensitivity to reward generally made independent contributions to personality-psychopathology factors. Sensitivity to reward made a substantial contribution to Externalizing (conduct problems, hyperactivity and inattention), whereas sensitivity to punishment contributed to Internalizing (shyness, fears and emotional symptoms). The link between sensitivity to punishment and Internalizing in older adolescents was stronger than in younger. The protective effect of punishment sensitivity on Externalizing was found in girls only. These findings demonstrate the role of motivational brain-behavior systems in the development of normal and abnormal patterns of behavior and emotions.
\end{abstract}

Keywords: personality, behavioral problems, emotional problems, psychopathology, reinforcement sensitivity, hierarchical structure, children, adolescents. 


\section{References}

Bergen, S. E., Gardner, C. O., \& Kendler, K. S. (2007). Age-related changes in heritability of behavioral phenotypes over adolescence and young adulthood: a meta-analysis. Twin Research and Human Genetics, 10(3), 423-433. doi:10.1375/twin.10.3.423

Bijttebier, P., Beck, I., Claes, L., \& Vandereycken, W. (2009). Gray's Reinforcement Sensitivity Theory as a framework for research on personality-psychopathology associations. Clinical Psychology Review, 29(5), 421-430. doi:10.1016/j.cpr.2009.04.002

Caspi, A., \& Shiner, R. L. (2008). Temperament and personality. In M. Rutter, D. Bishop, D. Pine, S. Scott, J. Stevenson, E. Taylor, \& A. Thapar (Eds.), Rutter's child and adolescent psychiatry (5th ed., pp. 182-198). London: Blackwell.

Chess, S. \& Thomas, A. (1984). Origins and evolution of behavior disorders. From infancy to early adult life. New York: Brunner/Mazel.

Colder, C. R., Trucco, E. M., Lopez, H. I., Hawk Jr, L. W., Read, J. P., Lengua, L. J., ... Eiden, R. D. (2011). Revised reinforcement sensitivity theory and laboratory assessment of BIS and BAS in children. Journal of Research in Personality, 45, 198-207. doi:10.1016/j.jrp.2011.01.005

Corr, P. J. (Ed.). (2008). The reinforcement sensitivity theory of personality. Cambridge: Cambridge University Press.

Corr, P. J., DeYoung, C. G., \& McNaughton, N. (2013). Motivation and personality: A neuropsychological perspective. Social and Personality Psychology Compass, 7(3), 158-175. doi:10.1111/spc3.12016

De Pauw, S. S., \& Mervielde, I. (2010). Temperament, personality and developmental psychopathology: A review based on the conceptual dimensions underlying childhood traits. Child Psychiatry and Human Development, 41, 313-329. doi:10.1007/s10578-009-0171-8

DeYoung, C. G. (2006). Higher-order factors of the Big Five in a multi-informant sample. Journal of Personality and Social Psychology, 91, 1138-1151. doi:10.1037/0022-3514.91.6.1138

Digman, J. M. (1997). Higher-order factors of the Big Five. Journal of Personality and Social Psychology, 73, 1246-1256. doi:10.1037/0022-3514.73.6.1246

Dirks, M. A., De Los Reyes, A., Briggs Gowan, M., Cella, D., \& Wakschlag, L. S. (2012). Annual Research Review: Embracing not erasing contextual variability in children's behavior-theory and utility in the selection and use of methods and informants in developmental psychopathology. Journal of Child Psychology and Psychiatry, 53, 558-574. doi:10.1111/j.1469-7610.2012.02537.x

Durbin, C. E., \& Hicks, B. M. (2014). Personality and psychopathology: A stagnant field in need of development. European Journal of Personality, 28, 362-386. doi:10.1002/per.1962

Erdle, S., \& Rushton, J. P. (2010). The general factor of personality, BIS-BAS, expectancies of reward and punishment, self-esteem, and positive and negative affect. Personality and Individual Differences, 48(6), 762-766. doi:1016/j.paid.2010.01.025

Fischer, R., \& Fontaine, J. R. J. (2011). Methods for investigating structural equivalence. In D. Matsumoto \& F. J. R. van de Vijver (Eds.), Cross-cultural research methods in psychology (pp. 179 215). New York: Cambridge University Press.

Goldberg, L. R. (2006). Doing it all bass-ackwards: The development of hierarchical factor structures from the top down. Journal of Research in Personality, 40(4), 347-358. doi:10.1016/j.jrp.2006.01.001 
Goodman, R., Slobodskaya, H. R., \& Knyazev, G. G. (2005). Russian child mental health: a cross-sectional study of prevalence and risk factors. European Child and Adolescent Psychiatry, 14(1), 28 33. doi:10.1007/s00787-005-0420-8

Gray, J. A. (1973). Causal models of personality and how to test them. In J. R. Royce (Ed.), Multivariate analysis and psychological theory (pp. 409-463). London: Academic Press.

Hirshfeld-Becker, D. R., Biederman, J., Calltharp, S., Rosenbaum, E. D., Faraone, S. V., \& Rosenbaum, J. F. (2003). Behavioral inhibition and disinhibition as hypothesized precursors to psychopathology: Implications for pediatric bipolar disorder. Biological Psychiatry, 53(11), 985-999. doi:10.1016/S0006-3223(03)00316-0

Hundt, N. E., Kimbrel, N. A., Mitchell, J. T., \& Nelson-Gray, R. O. (2008). High BAS, but not low BIS, predicts externalizing symptoms in adults. Personality and Individual Differences, 44(3), 565-575. doi:1016/j.paid.2007.09.018

Knyazev, G. G., Zupan i , M., \& Slobodskaya, H. R. (2008). Child personality in Slovenia and Russia. Structure and mean level of traits in parent and self-ratings. Journal of Cross-Cultural Psychology, 39(3), 317-334. doi:10.1177/0022022108314542

Krueger, R. F., \& Markon, K. E. (2006). Reinterpreting comorbidity: A model-based approach to understanding and classifying psychopathology. Annual Review of Clinical Psychology, 2, 111-133. doi:10.1146/annurev.clinpsy.2.022305.095213

Krueger, R. F., \& Tackett, J. L. (2003). Personality and psychopathology: Working toward the bigger picture. Journal of Personality Disorders, 17, 109-128. doi:10.1521/pedi.17.2.109.23986

Kuznetsova, V. B., \& Slobodskaya, E. R. (2010). Sensitivity to punishment and reward questionaire in children. Psikhologicheskii Zhurnal, 31(2), 104-113. (in Russian)

Kuznetsova, V. B., Slobodskaya, E. R., \& Rippinen, T. O. (2013). Adaptation of Sensitivity to Punishment and Sensitivity to Reward Questionnaire in adolescents. Psikhologicheskii Zhurnal, 34(1), 97-108. (in Russian)

Lahey, B. B., Rathouz, P. J., Van Hulle, C., Urbano, R. C., Krueger, R. F., Applegate, B., ... Waldman, I. D. (2008). Testing structural models of DSM-IV symptoms of common forms of child and adolescent psychopathology. Journal of Abnormal Child Psychology, 36, 187-206. doi:10.1007/s10802007-9169-5

Markon, K. E., Krueger, R. F., \& Watson, D. (2005). Delineating the structure of normal and abnormal personality: An integrative hierarchical approach. Journal of Personality and Social Psychology, 88 , 139-157. doi:10.1037/0022-3514.88.1.139

O'Connor, B. P. (2000). SPSS and SAS programs for determining the number of components using parallel analysis and Velicer's MAP test. Behavior Research Methods, Instruments and Computers, 32(3), 396-402. doi:10.3758/BF03200807

Pavlov, I. P. (1923). Dvadtsatiletnii opyt ob»ektivnogo izucheniya vysshei nervnoi deyatel'nosti (povedeniya) zhivotnykh [Twenty years experience of objective research on higher nervous activity]. Moscow/Petrograd: Gosizdat.

Rutter, M., Bishop, D., Pine, D., Scott, S., Stevenson, J., Taylor, E., \& Thapar, A. (Eds.). (2008). Rutter's child and adolescent psychiatry (5th ed.). Oxford: Blackwell.

Slobodskaya, H. R. (2011). Two superordinate personality factors in childhood. European Journal of Personality, 25, 453-464. doi:10.1002/per.810

Slobodskaya, H. R. (2014). The hierarchical structure of personality and common psychopathology in childhood. Journal of Research in Personality, 53, 36-46. doi:10.1016/j.jrp.2014.08.005 
Slobodskaya, H. R., \& Zupančič, M. (2010). Development and validation of the Inventory of Child Individual Differences - Short version in two Slavic countries. Studia Psychologica, 52, 23-39.

Tackett, J. L. (2006). Evaluating models of the personality-psychopathology relationship in children and adolescents. Clinical Psychology Review, 26(5), 584-599. doi:10.1016/j.cpr.2006.04.003

Tackett, J. L., Slobodskaya, H. R., Mar, R. A., Deal. J., Halverson, Ch. F. Jr., Baker, S., ... Besevegis., E. (2012). The hierarchical structure of childhood personality in five countries: Continuity from early childhood to early adolescence. Journal of Personality, 80(4), 847-879. doi:10.1111/j.14676494.2011.00748.x

Tremblay, R. E. (2010). Developmental origins of disruptive behaviour problems: The original sin hypothesis, epigenetics and their consequences for prevention. Journal of Child Psychology and Psychiatry, 51(4), 341-367. doi:10.1111/j.1469-7610.2010.02211.x

van der Heijden, P. T., Rossi, G. M. P., van der Veld, W. M., Derksen, J. J. I., \& Egger, J. I. M. (2013). Personality and psychopathology: Higher order relations between the Five Factor Model of personality and the MMPI-2 Restructured Form. Journal of Research in Personality, 47(5), 572-579. doi:10.1016/j.jrp.2013.05.001

Helena R. Slobodskaya - associate professor, senior research fellow, Federal State Budgetary Scientific Institution «Scientific Research Institute of Physiology and Basic Medicine», D.Sc. Research area: personality development, child mental health.

E-mail: hslob@physiol.ru 